\title{
Paradigmenwechsel der Werte
}

\section{Rouven Porz}

Prof. Dr., Medizinethik und ärztliche Weiterbildung, Insel Gruppe, Inselspital Bern, Mitglied der Redaktion Ethik der SÄZ

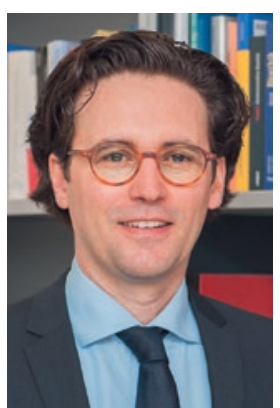

In den letzten Monaten wurden viele von unseren sonst so selbstverständlichen Freiheitsrechten eingeschränkt. Wir können nicht mehr frei reisen, wir können uns nicht mehr mit so vielen Leuten treffen, wie wir möchten, wir können uns bei Treffen im Freundes- und Familienkreis nicht mehr frei umarmen und küssen, der Besuch von Grossveranstaltungen wie Musikfestivals oder Sportereignissen ist praktisch unmöglich. Einschränkungen, Freiheitsbeschränkungen auf Kosten unserer Selbstbestimmung. Tatsächlich, das sind wir nicht gewohnt. Gerade in der Schweiz ist die Selbstbestimmung ein wichtiger Wert, vielleicht sogar der wichtigste im gegenseitigen Miteinander. Aber jetzt hat durch die COVID-19-Pandemie ein Wertewechsel stattgefunden, gewissermassen ein Paradigmenwechsel der Werte. Wir mussten lernen, dass es neben der Selbstbestimmung noch einen weiteren wichtigen Wert gibt. Diesen Wert kann man in zwei Facetten ausdrücken. Die Rede ist erstens von der öffentlichen Gesundheit (Public Health) bzw. zweitens von der Solidarität, die es unter den Einzelnen braucht, um in gegenseitigem Einverständnis diese öffentliche Gesundheit nicht weiter zu gefährden. Wir reden von einem wahrgenommenen Wertekonflikt zwischen Selbstbestimmung und Solidarität. Wir sehen diesen Konflikt jeden Tag in den Medien. Auf der einen Seite der Appell zum Gemeinsamen, auf der anderen Seite das Beharren auf persönlicher "Freiheit». Schwierig. Ja, es ist sehr schwierig. Denn Wertekonflikte sind immer schwierig, sie gehen mit unterschiedlichen Meinungen einher, oft mit Schwarz und Weiss. Und normalerweise können wir diesen Konfrontationen geschickt aus dem Weg gehen, unseren eigenen gewohnten Beschäftigungen nachgehen (Selbstbestimmung!), aber diesmal gibt es kein richtiges Entkommen. Wir sitzen ja irgendwie alle im selben Boot (Solidarität!).

Es gibt aber auch versöhnliche Gedanken zu diesem Paradigmenwechsel. Erstens müssen wir anerkennen, dass unterschiedliche Menschen unterschiedliche Werte haben. Das muss nicht schlecht sein. Zweitens ist es durchaus möglich, Schwarz und Weiss zu einem schönen Grau zu mischen. Es gibt also auch Wege, die
Selbstbestimmung zu würdigen und trotzdem solidarisch zu handeln. Und drittens, und hier können wir uns lose des wohl bekanntesten Philosophen der Aufklärung bedienen, Immanuel Kant (1724-1804), der wohl sagen würde: «Freiheit» bedeutet nicht, Rechte zu haben, sondern dass man Pflichten hat [1]. Gerade dieser Gedanke Kants bringt uns zu einem neuen Paradigmenwechsel. Vielleicht leben wir im Moment tatsächlich in einer Zeit, in der wir über unsere humanitären Pflichten nachdenken sollten, anstatt nur Persönlichkeitsrechte einzufordern. Ich zumindest empfinde dies als eine versöhnliche Aufforderung und Idee.

Und auch meine Disziplin der Medizinethik wird sich diesem Paradigmenwechsel stellen müssen. Das vorrangige medizinethische Prinzip der Patientenautonomie tritt aktuell etwas in den Hintergrund gegenüber dem Public Health-Gedanken. Gut so, denn das wird

\section{Wir sollten über unsere humanitären Pflichten} nachdenken, anstatt nur Persönlichkeitsrechte einzufordern.

auch unsere Disziplin noch mehr dazu zwingen, intensiver darüber nachzudenken, wie scheinbar konkurrierende Wertesysteme parallel existieren und funktionieren können. Wir werden weiterhin den Respekt vor der Patientenautonomie als extrem wichtigen Wert sehen, aber vielleicht nicht mehr als den immer und allein vorrangigen. Und auch der Wert der Solidarität könnte endlich stärkeren Einzug ins medizinethische Denken halten. Vielversprechende Denkanstösse und Vorarbeiten dazu gibt es schon [2]. Vielleicht werden wir sogar den Disziplinbegriff ändern müssen; vielleicht ist es an der Zeit, den etwas engen Begriff der «Medizinethik» zu einer grösser denkenden «Health Care Ethics» zu erweitern.

\section{Literatur}

1 Immanuel Kant. Die Metaphysik der Sitten. Original 1797 [Kapitel: Einleitung in die Rechtslehre].

2 Ruud ter Meulen. Solidarity and Justice in Health and Social Care. Cambridge University Press 2017. Oder: Barbara Prainsack und Alena Buyx. Solidarity in Biomedicine and Beyond. Cambridge University Press 2017. 